\title{
Multiaxial Inverse Stress Analysis for Indentation Creep
}

\author{
Masao Sakane ${ }^{1,1}$, Akihiko Hirano ${ }^{2}$, Naomi Hamada ${ }^{3}$, Yukari Hoya ${ }^{1}$, Takahiro Oka $^{1}$, \\ Masataka Furukawa ${ }^{1}$ and Takamoto Itoh $^{1}$ \\ ${ }^{1}$ Ritsumeikan University, 1-1-1 Nojihigashi Kusatsu Shiga, 525-8577, Japan \\ ${ }^{2}$ Osaka Sangyo University, 3-1-1 Nakagaichi Daito, Osaka, 574-8530, Japan \\ ${ }^{3}$ Hiroshima Kokusai Gakuin University, 6-20-1 Nakano Akiku Hiroshima, Hiroshima, 739-0321, \\ Japan
}

\begin{abstract}
This paper presents an extracting method of creep exponent and coefficient in Norton's law from an indentation creep test analytically, assisted by a finite element analysis. Previous studies extracted only the creep exponent empirically with no theoretical verification. No extracting method on the creep coefficient has been proposed from an indentation creep test. By analyzing multiaxial stress states under a ball indenter in combination of a creep constitutive equation, an analytical method to derive the creep coefficient is developed. The method is verified to have a satisfactorily accuracy to determine the two material constants in Norton's law by finite element analyses and in experiments.
\end{abstract}

\section{Introduction}

This paper proposes an extracting method of creep exponent and coefficient in Norton's law from an indentation creep test. Indentation tests have been widely used to obtain mechanical properties of materials. A recent topic of indentation studies is to extract material constants from hardness testing, like Young's modulus, strain hardening exponent and coefficient etc. Among these studies, hardness studies to extract creep exponent and coefficient have attracted interests of researchers and many studies challenge this topic.

These extracting studies reported that the creep exponent extracted from an indentation creep test agreed with that in uniaxial creep tests [1,2], but a theoretical background of the agreement has not been proved. In addition to this, no theoretical method to obtain the creep coefficient has been reported from an indentation creep test. Coefficient obtained from indentation creep tests were smaller than those from uniaxial creep tests. The authors have examined the reason for the deviation of the coefficient between the two tests and have found that all the previous studies [3,4] did not take account of stress multiaxiality under an indenter.

The objective of this paper is to develop a new method of extracting creep exponent and coefficient from an indentation creep test. Multiaxial stress states under an indenter in creep

\footnotetext{
${ }^{1}$ Corresponding author: sakanem@se.ritsumei.ac
} 
deformation are analyzed by an elastic-creep finite element method (FEM) with variating the two material constants widely and an empirical formula to expressing multiaxial stresses under an indenter is derived. Using an inelastic creep constitutive equation in combination of the derived multiaxial stresses, a method of extracting the creep exponent and coefficient is proposed. Creep exponents and coefficients derived by this method are compared with those provided as the input data to the FEM analysis. A new indentation creep machine is developed to examine the validity of the new method experimentally. The creep exponents and coefficients obtained by the proposed indentation test method are compared with those in uniaxial creep tests for a $\mathrm{Sn}-37 \mathrm{~Pb}$ eutectic solder.

\section{Finite element analysis}

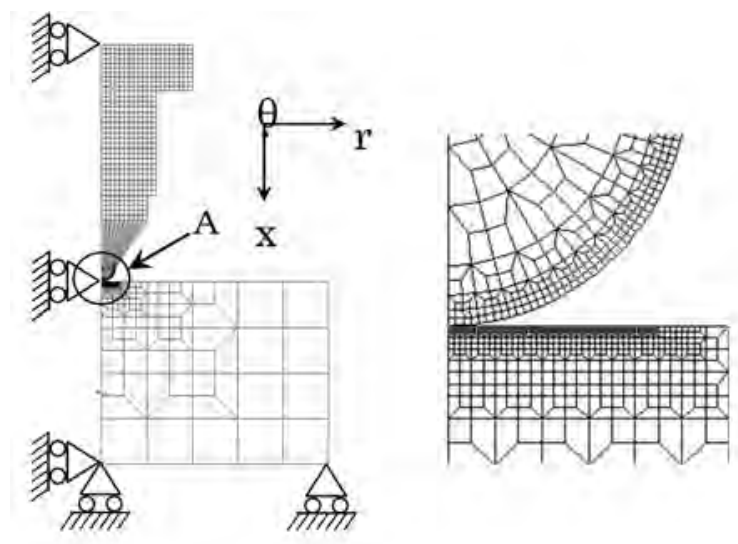

Fig.1. Finite element mesh of an indenter and a specimen.

This study performs finite element analyses to simulate the indentation creep tests using the finite element mesh shown in Fig.1. The figure also shows the coordinate system employed in this study. The origin of the coordinate system is set to the tip of the indenter ball. The model consists of a specimen, an indentation ball with a $0.79 \mathrm{~mm}$ radius and a ball holder. The element type is of an axisymmetric iso-parametric 4-node element. The numbers of elements and nodes of the model are 1603 and 1765, respectively. The constraint condition is to fix axisymmetric line of the model in the $r$-direction and the bottom line of the specimen in the $x$-direction.

Norton type creep constitutive equation was used to relate equivalent creep strain rates to equivalent stresses as expressed below.

$$
\dot{\bar{\varepsilon}}=\alpha \bar{\sigma}^{\beta},
$$

where $\dot{\bar{\varepsilon}}$ is the von Mises equivalent creep strain rate $(\mathrm{mm} / \mathrm{h}), \alpha$ the creep coefficient, $\bar{\sigma}$ the von Mises equivalent stress (MPa) and $\beta$ the creep exponent. Elastic-creep finite element analyses were performed using the Young's modulus of $150 \mathrm{GPa}$, and stresses and strains under the indenter and the $x$-directional displacement of the holder top were output with time. The combination of $\alpha$ and $\beta$ analyzed is tabulated in Table 1. The value of $\alpha$ was ranged from $10^{-12}$ to $10^{-20}$ and $\beta$ from 3 to 11 . These ranges of creep exponents and coefficients analyzed mostly cover $\alpha$ and $\beta$ values of high temperature materials in practical applications. 
Table 1 Creep exponent and coefficient used in FEM analyses.

\begin{tabular}{|c|c|c|c|c|c|}
\hline$\beta$ & $10^{-12}$ & $10^{-14}$ & $10^{-16}$ & $10^{-18}$ & $10^{-20}$ \\
\hline 3 & $\bigcirc$ & $\bigcirc$ & $\bigcirc$ & $\bigcirc$ & $\bigcirc$ \\
\hline 5 & $\bigcirc$ & $\bigcirc$ & $\bigcirc$ & $\bigcirc$ & $\bigcirc$ \\
\hline 7 & $\bigcirc$ & $\bigcirc$ & $\bigcirc$ & $\bigcirc$ & $\bigcirc$ \\
\hline 9 & $\bigcirc$ & $\bigcirc$ & $\bigcirc$ & $\bigcirc$ & $\bigcirc$ \\
\hline 11 & $\bigcirc$ & $\bigcirc$ & $\bigcirc$ & $\bigcirc$ & $\bigcirc$ \\
\hline
\end{tabular}

\section{Theoretical approach to determine creep exponent and coefficient}

\subsection{Stress-strain rate relationship in indenter creep testing}

From the geometric relationship, the projected area of the contact surface of indenter $(A)$ to specimen is expressed by the following equation with the ball radius $(r)$ and current indentation depth $(h)$ as

$$
A=\pi h(2 r-h)
$$

By using the above equation, the indentation stress $\left(\sigma_{\text {Ind }}\right)$ under indenter ball can be evaluated by the following equation

$$
\sigma_{\text {Ind }}=\frac{P}{A}=\frac{P}{\pi h(2 r-h)},
$$

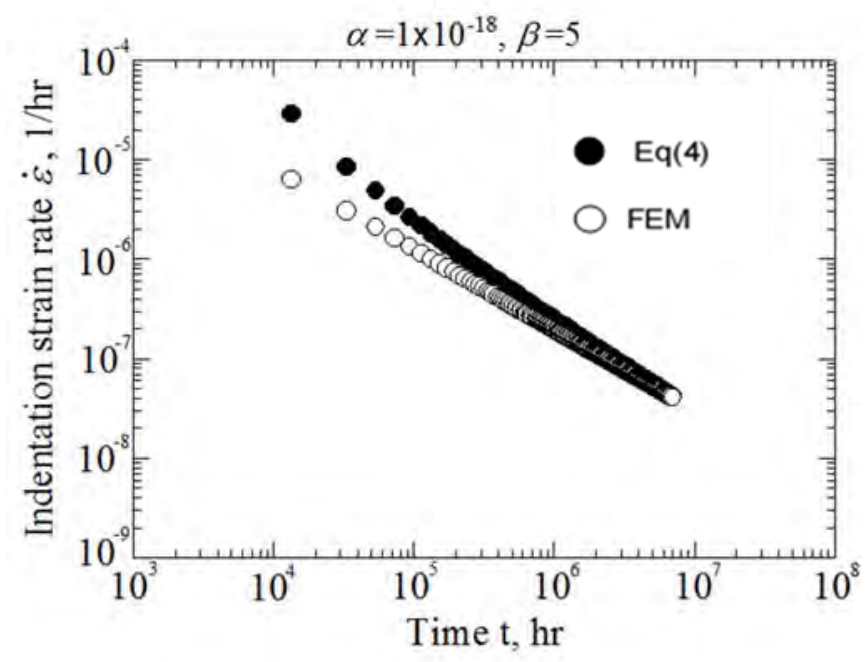

Fig.2. Comparison of indentation creep strain rates with strain rates of a finite element under indenter calculated by FEM. 
where $P$ is the load applied to the indenter. The direction of the indentation stress evaluated by Eq.(3) is the $x$-direction, so the in-dentation stress is identical to the $x$-directional stress. The strain rate of testing material under the ball indenter from the indentation creep test (indentation strain rate) $\left(\dot{\varepsilon}_{\text {Ind }}\right)$ is calculable from the following equation $[5,6]$.

$$
\dot{\varepsilon}_{\text {Ind }}=\frac{1}{h} \frac{d h}{d t} .
$$

To confirm the validity of Eq.(4), strain rates calculated by Eq.(4) are compared with those obtained by the finite element analysis in Fig. 2 for a typical case of $\alpha=1 \times 10^{-18}$ and $\beta=5$. In this comparison, the indentation strain rates calculated by Eq.(4) using $h$ obtained by FEM are compared with the strain rates of the element at $r=0$ and just one element away in the $x$-direction from the indenter ball tip obtained by FEM (finite element strain rate). Eq.(4) overestimates the creep strain rate in the short term region up to $10^{5} \mathrm{hs}$ but the indentation strain rate approaches to the finite element strain rate as time increases. After $10^{6} \mathrm{hs}$ in this case, the indentation strain rates closely agree with the finite element strain rates with a satisfactory accuracy. Almost the same trend was also found in the other combinations of creep exponents and coefficients shown in Table 1 analyzed. Therefore, the strain rate calculated by Eq.(4) as well as the stress amplitude estimated by Eq.(3) have a reasonable accuracy to estimate those under the indenter ball, whereas the other cases are not graphically presented from the reason of the paper space.

Fig. 3 compares the indentation strain rate-indentation stress relationship obtained by Eq.(3) and (4) with the uniaxial creep relationship input to FEM analysis. The slope in the

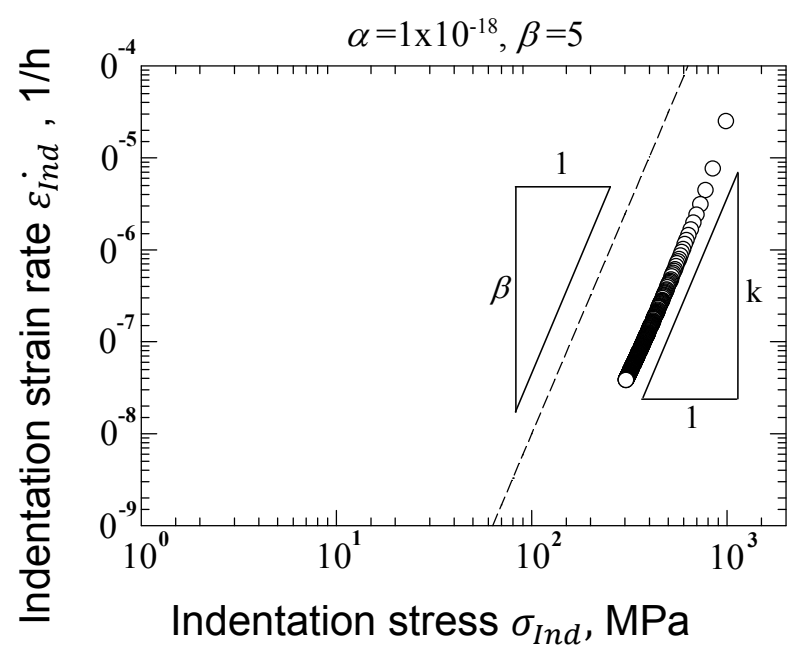

Fig.3. Comparison of the indentation strain rate-the $\mathrm{x}$-directional stress relationship derived by Eq.(3) and (4) with the uniaxial creep relationship input to FEM analysis.

former relationship is denoted as $k$ and that in the latter relationship as $\beta$. The slope obtained from the indentation test is $k=5$ and that input to the analysis is also $\beta=5$. The both values closely agree each other and such agreement has been already reported in the literature $[1,2]$. However, the indentation creep strain rates are smaller than the uniaxial creep strain rate at the same stress by about two orders of magnitude. This means that the creep coefficient $\alpha$ obtained from the indentation test is smaller than the uniaxial one. 


\subsection{Theoretical derivation of creep exponent and coefficient from indentation creep test}

The disagreement of the creep coefficient between the indentation test and the uniaxial creep test results from that Eq.(2)-(4) do not take account of the stress multiaxiality under the indenter ball. Creep deformation behavior is strongly dependent on the stress multiaxiality so that it should be taken account of in the equations for extracting the coefficient from an indentation test.

Fig. 4 depicts the variation of the stress ratios with indenter displacement for six $\beta$ values and $\alpha=1.8 \times 10^{-18}$ obtained from the FEM analyses. In the figure, the stress ratios of $w_{r}$ and $w_{\theta}$ are the ratios of the radial stress and the tangential stress to the $\mathrm{x}$-directional stress, respectively, i.e.,

$$
w_{r}=\frac{\sigma_{r r}}{\sigma_{x x}} \quad w_{\theta}=\frac{\sigma_{\theta \theta}}{\sigma_{x x}},
$$

where $\sigma_{r r}$ is the radial principal stress and $\sigma_{\theta \theta}$ is the tangential principal stress under the indenter. The FEM model used in this study is the axisymmetric model so that the radial
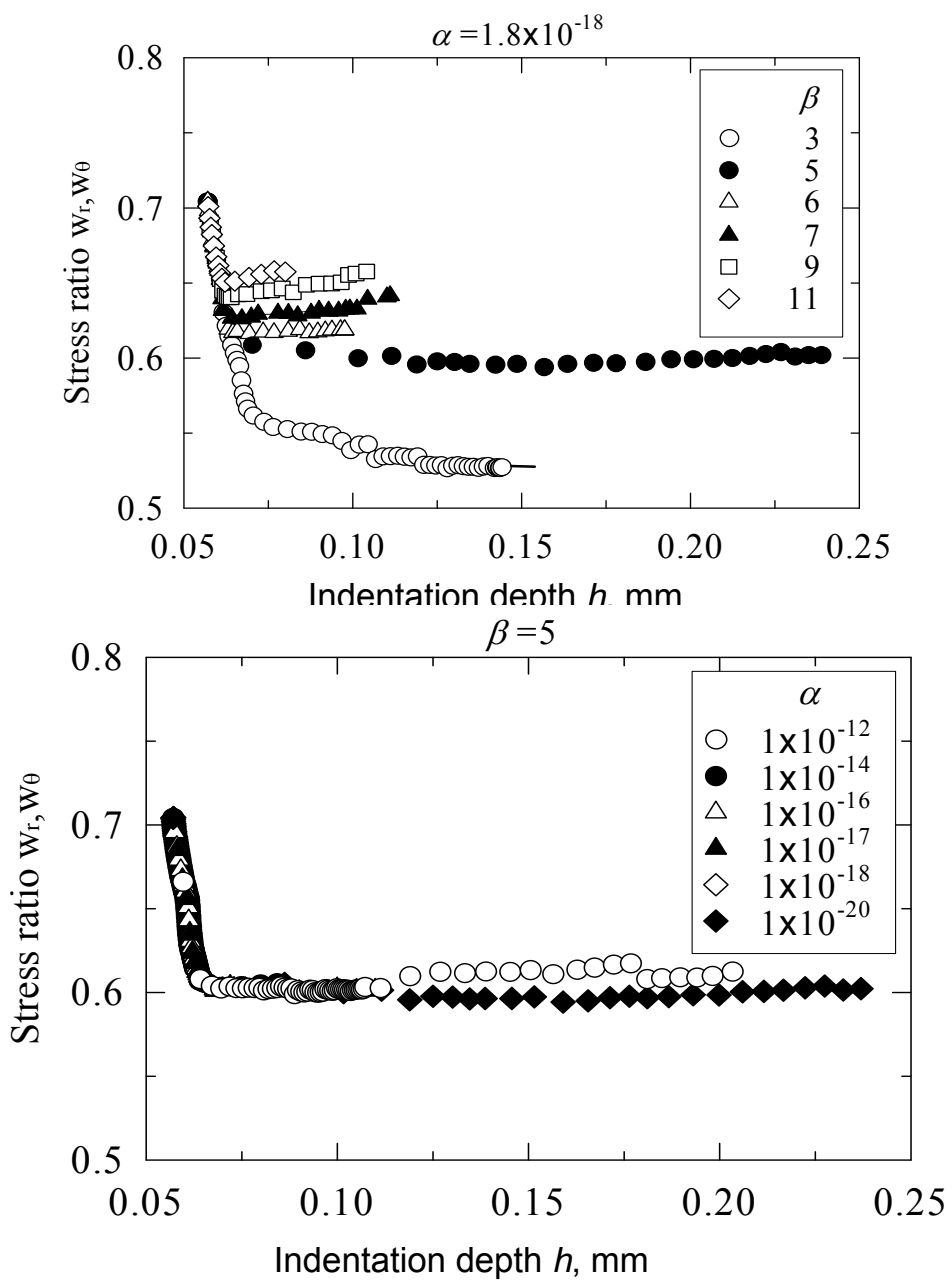

Fig.4. Reduction in stress ratio with indention depth. 
principal stress has the same amplitude as the tangential principal stress. The figure indicates that the stress ratios have the values around 0.7 immediately after the indentation test starts but they decrease as the indenter displacement increases depending on $\beta$.

Fig.5 illustrates the variation of the two stress ratios with indentation depth for six $\alpha$ values and $\beta=5$. The stress ratios take a stabilized value of around 0.6 in the indentation depth beyond $0.025 \mathrm{~mm}$ independent of the value of $\alpha$ in this case. Similar stabilized values were also confirmed for the other cases of $\beta$ with different stabilized stress ratios. The relationship between the stabilized stress ratio and $\beta$ is shown in Fig. 6. Variations of the stabilized stress ratios are expressed by a quadratic function written in the figure. From the relationship shown in Fig.6, the stabilized stress ratios can be determined from the creep exponent $\beta$ that is obtained from indentation creep tests. Since the x-directional stress can be calculated in the indentation creep tests by Eq.(3), we can obtain all three principal stresses under the indenter ball by using $\beta$ in the indentation test and the equation in Fig. 6.

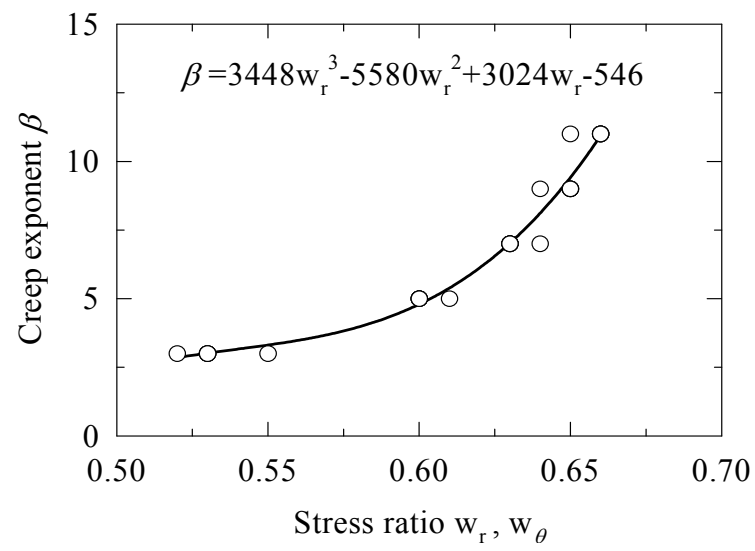

Fig.6. Dependence of the principal stress ratios on creep exponent.

Creep strain rates in the x-direction under multiaxial stress states $\left(\dot{\varepsilon}_{x x}\right)$ are related to von Mises stress $(\bar{\sigma})$ and deviatoric stress $\left(S_{x x}\right)$ expressed by the following equation from the potential theory of plasticity.

$$
\dot{\varepsilon}_{x x}=\frac{3}{2} \alpha \bar{\sigma}^{(\beta-1)} S_{x x}
$$

Von Mises equivalent stress and the $\mathrm{x}$-directional deviatoric stress in axisymmetric conditions are expressed by Eq.(7) and (8) with the principal stresses $\left(\sigma_{x x}, \sigma_{r r}, \sigma_{\theta \theta}\right)$.

$$
\begin{gathered}
\bar{\sigma}=\frac{1}{\sqrt{2}} \sqrt{\left(\sigma_{x x}-\sigma_{r r}\right)^{2}+\left(\sigma_{r r}-\sigma_{\theta \theta}\right)^{2}+\left(\sigma_{\theta \theta}-\sigma_{x x}\right)^{2}} . \\
S_{x x}=\sigma_{x x}-\frac{1}{3}\left(\sigma_{x x}+\sigma_{r r}+\sigma_{\theta \theta}\right) .
\end{gathered}
$$

Substituting $\sigma_{r r}$ and $\sigma_{\theta \theta}$ in Eq.(5) to Eq.(7), the equivalent stress ( $\left.\bar{\sigma}\right)$ can be expressed with only $\sigma_{x x}$ by setting $w_{r}=w_{\theta}=w$ as

$$
\bar{\sigma}=(1-w) \sigma_{x x}
$$

Similarly, the deviatoric stress in the x-direction is written with $\sigma_{x x}$ as 


$$
S_{x x}=\frac{2}{3}(1-w) \sigma_{x x}
$$

Combining Eq.(6), (9) and (10) gives

$$
\dot{\varepsilon}_{x x}=\alpha\left[(1-w) \sigma_{x x}\right]^{\beta} .
$$

Equation (11) is the equation that relates the $\mathrm{x}$-directional principal stress and $\mathrm{x}$-directional strain rate under the indenter. Using the x-directional indentation stress $\left(\sigma_{\text {Ind }}\right)$ in Eq.(3) and indentation strain rate ( $\left.\dot{\varepsilon}_{I n d}\right)$ in Eq.(4), the following equation is finally obtained.

$$
\dot{\varepsilon}_{\text {Ind }}=\alpha(1-w)^{\beta} \sigma_{\text {Ind }}^{\beta}
$$

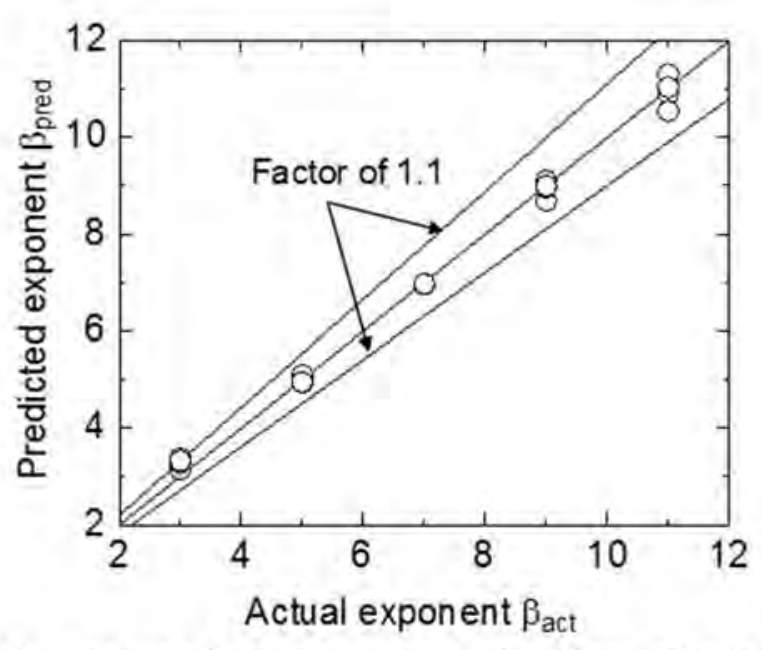

Fig. 7. Comparison of creep exponents derived from indentation tests with those in uniaxial creep tests in FEM analysis.

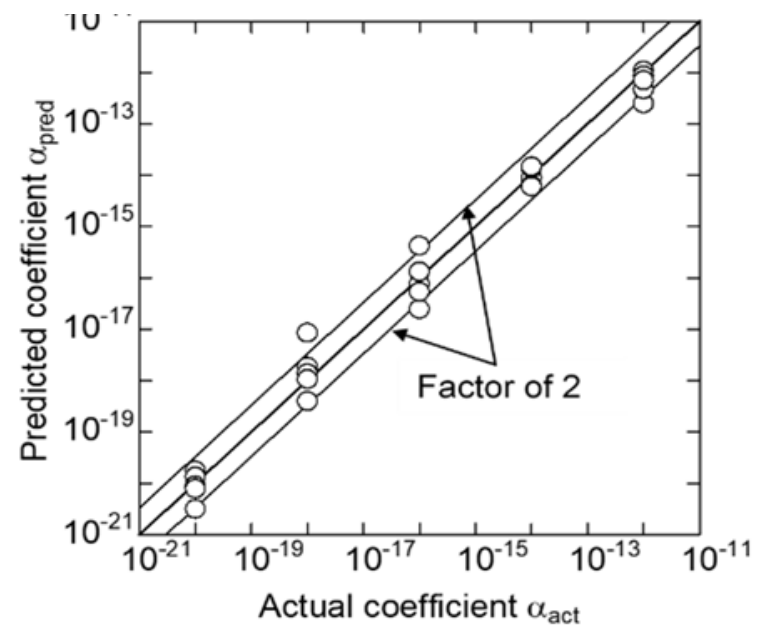

Fig 8. Comparison of creep coefficient derived from indentation tests with those in uniaxial creep tests in FEM analysis. 
The equation clearly demonstrates that the slope of the creep strain rate-stress relationship obtained in the indentation creep test by Eq.(12) is identical to the creep exponent in a uniaxial creep test. Therefore, we can use the slope obtained in the indentation test as that in the uniaxial test because the agreement of the slope between the indentation creep test and the uniaxial creep test is demonstrated by Eq.(12). The creep coefficient is also calculatable from Eq.(12) using the indentation strain rate $\left(\dot{\varepsilon}_{I n d}\right)$, the indentation stress $\left(\sigma_{I n d}\right)$, the stress ratio $(w)$ and $\beta$ obtained in the indentation test.

Creep exponents and coefficients obtained using Eq.(12) in FEM indentation simulations with the material constants shown in Table 1 are compared with those in uniaxial creep data input into FEM analysis in Fig.7 and 8, respectively. Creep exponents derived from the indentation simulation agree with those in uniaxial creep data input to FEM analysis within a factor of 1.1, Fig.7. Also, estimated creep coefficients agree with those in the uniaxial creep data within a factor of two, Fig.8. Therefore, it is concluded that the extraction method proposed in this paper has a satisfactory accuracy to estimate creep exponents and coefficients.

\section{Experimental approach to extract creep exponent and coefficient}

To confirm the validity of the proposed method of extracting creep exponent and coefficient experimentally, a new indentation creep test machine was developed for solders. The schematic of the indentation creep test machine is shown in Fig.9. A dead weight system is employed as a loading device and indentation depth was measured by a LVTD connected to the loading rod. The temperature of a specimen was raised by a furnace with small ceramic heaters attached on the inner wall of the furnace. The radius of the indenter is $0.79 \mathrm{~mm}$.

Fig. 10 illustrates indentation depth with time for a Sn-37Pb eutectic solder at $313 \mathrm{~K}$ at $2.5 \mathrm{~N}$, $5.0 \mathrm{~N}$ and $10.0 \mathrm{~N}$. Shapes of the three curves are similar to the creep curve of conventional heat resistant steels consisting of initial and secondary creep stages. Creep strain rates are faster in the initial stage after loading but they decrease with time. The contacted area

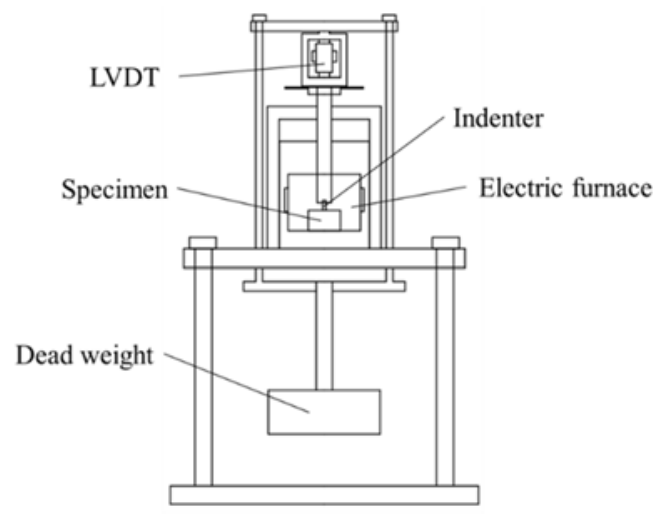

Fig.9. Schematic of an indentation creep tester for solders. 


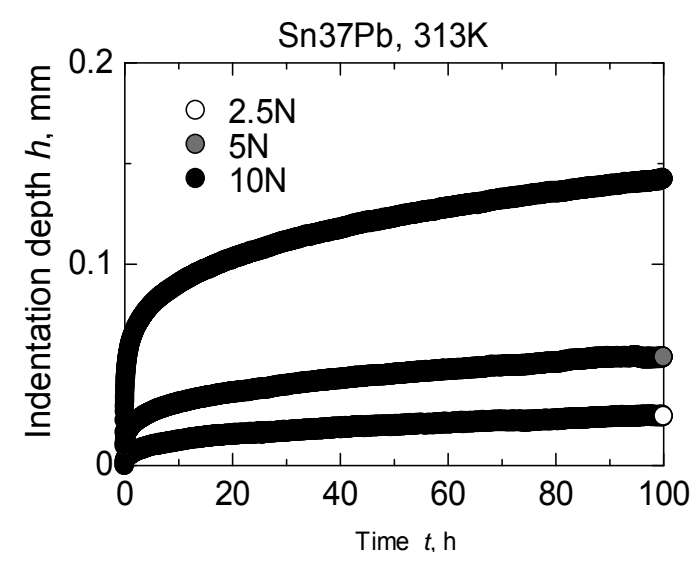

Fig.10. Indentation depth-time relationship for $\mathrm{Sn}-37 \mathrm{~Pb}$ solder at $313 \mathrm{~K}$.

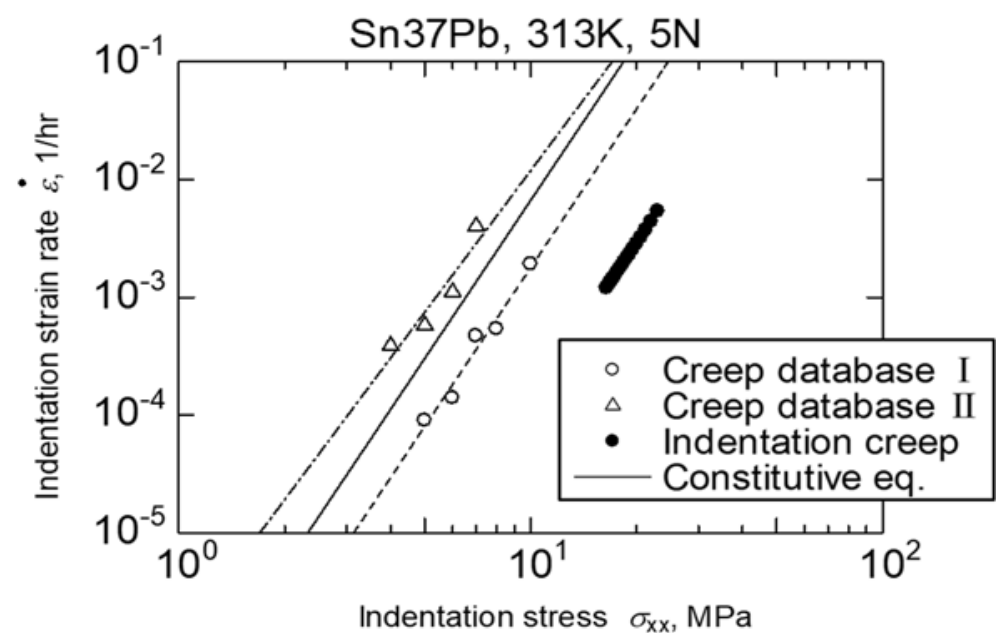

Fig.11. Comparison of creep strain rates between indentation test and uniaxial creep tests for $\mathrm{Sn}-37 \mathrm{~Pb}$ solder at $313 \mathrm{~K}$.

between the indenter and specimen is smaller in the initial stage resulting in higher stress but the contacted area increases with time resulting in the reduction in stress.

Using the data of $5 \mathrm{~N}$ in Fig.10, indentation stresses-indentation creep strain rates are plot in Fig. 11 with solid dots. Also, uniaxial tensile creep data are plot with triangles and open circles[7,8]. The two kinds of uniaxial tensile data are produced from the same material, but heat batches are different. Since the tensile solder specimens were made by casting, microstructures of the two batches are presumably somewhat different yielding the different creep strain rates in Fig.11. The difference in creep strain rate between the two batches, however, is in the range of acceptable scatter.

The slope of the indentation creep data with solid dots in the figure is very close to that of the uniaxial creep data but the strain rates in the indentation test are smaller than those in uniaxial creep tests which means that the creep coefficient by the indentation test is smaller than that in uniaxial tests. The reason of the smaller creep coefficient results from the multiaxial stresses under the indenter and this study developed a new method to extract the creep coefficient expressed in Eq.(12). Extracted creep strain rates are shown with the solid line in Fig.11. The extracted strain rates locate between the two uniaxial strain rates of the 
uniaxial data. Considering that the creep exponents and creep coefficients extracted from the indentation tests agree with those in uniaxial creep tests by FEM and in experiments, the extracting method developed in this study conveys a satisfactory reliability for a practical use.

\section{Conclusions}

1. A new method of extracting creep exponent and coefficient from indentation creep tests is proposed with the assistance of extensive finite element creep indentation analyses. Creep exponents and coefficient extracted by the new method agree well with those in uniaxial creep tests input to finite element analysis.

2. A new indentation creep test machine was developed to confirm the validity of the new extracting method of creep exponent and coefficient for solders. Creep exponent and coefficient of a Sn-37Pb extracted in the indentation method agree well with those obtained in uniaxial tests.

\section{References}

1. S.N.G. Chu, J.C.M. Li, Impression creep; a new creep test, J. Mater. Sci. $12: 2200$ 2208(1977).

2. V. Raman, R. Berriche, An investigation of the creep processes in tin and aluminum using a depth-sensing indentation technique, J. Mater. Res. 7 : 627-638(1992).

3. N.Q. Chinh, P. Szommer, Mathematical description of indentation creep and its application for the determination of strain rate sensitivity, Mater. Sci. Eng. A. 611: 333-336 (2014).

4. M. Kim, K.P. Marimuthu, S. Jung, H. Lee, Contact size-independent method for estimation of creep properties with spherical indentation, Comput. Mater. Sci. 113: 211-220(2016).

5. M.J. Mayo, W.D. Nix, A micro-indentation study of superplasticity in $\mathrm{Pb}, \mathrm{Sn}$, and $\mathrm{Sn}$ 38 wt\% Pb, Acta Metall. 36: 2183-2192(1988).

6. W.H. Poisl, W.C. Oliver, B.D. Fabes, The relationship between indentation and uniaxial creep in amorphous selenium, J. Mater. Res. 10: 2024-203 2(1995).

7. Factual Database on Creep and Creep-Fatigue Properties of Sn-37Pb and Sn-3.5Ag Solders, Japan Society of Materials Science, (2004).

8. Factual Database on Tensile, Creep, Low Cycle Fatigue and Creep-fatigue of Lead and Lead-free Solders, Japan Society of Materials Science, (2013). 